\title{
Publicaciones científicas de pregrado en Medicina: Caso de una Universidad de Lambayeque
}

\author{
Undergraduate Scientific Publications in Medicine: Case of a Lambayeque University
}

\section{Sr. Editor:}

En la actualidad es evidente que enseñar investigando desde la etapa de pregrado, beneficia en muchos aspectos la tarea de formar profesionales técnica y éticamente responsables con las demandas sociales. Entre los diversos impactos que genera esta práctica se encuentra la evaluación para los rankings de publicación internacional como la de SCImago, que evalúa la actividad investigadora de las Instituciones de Educación Superior en Iberoamérica, tomando en cuenta el número de publicaciones en revistas médicas indizadas en la base de datos Scopus ${ }^{\circledR}$ de la editorial Elsevier que contiene artículos en ciencias, medicina, tecnología, ciencias sociales, artes y humanidades (1).

Si bien es cierto, investigar resulta fundamental para el desarrollo intelectual de la persona, todo el proceso deberá concluir en la elaboración de un producto, el mismo que es la evidencia del trabajo realizado. Se conoce que a nivel latinoamericano existe un bajo porcentaje de publicaciones de investigación por parte de los estudiantes de medicina (2), y entre las numerosas causas que se han encontrado en estudios a nivel continental relacionados con el factor estudiante podríamos resaltan con mayor frecuencia que no investigan porque carecen de tiempo y porque no reciben la suficiente información para concretar la publicación de sus estudios, es decir no son instruidos y no cuentan con el soporte para finiquitar el proceso de investigación de parte de las universidades $(2,3)$
De acuerdo a la nueva Ley Universitaria $\mathrm{N}^{\circ} 30220$ (4) promulgada en el año 2014 en el Perú, se genera el marco normativo donde se menciona la creación de la Superintendencia Nacional de Educación Superior Universitaria (SUNEDU), la cual tiene la finalidad de "verificar el cumplimiento de condiciones básicas de calidad (CBC) para ofrecer el servicio educativo superior universitario y autorizar el funcionamiento de las universidades". En relación con la investigación, la Universidad debe tener un Vicerrectorado de Investigación u órgano de gobierno similar, contar con líneas de investigación aprobadas, presupuesto, equipamiento, personal dedicado a investigación, registro de proyectos de investigación, código de ética, protección de propiedad intelectual, docentes registrados en el Directorio Nacional de Investigadores e Innovadores del CONCYTEC (https://dina.concytec. gob.pe) (5). Todo esto, sin duda nos ofrece un mejor panorama para institucionalizar la ruta de generar conocimiento a través de la ciencia e investigación, con una marcada tendencia de incrementar la producción científica de las universidades.

En este sentido es válido el esfuerzo para motivar y brindar todas las facilidades necesarias para que los estudiantes de pregrado de las escuelas de medicina puedan recorrer todo el proceso de la investigación científica y culminar en la publicación de los resultados mediante el articulo científico el mismo que buscara una revista indizada de una base de datos de prestigio local e internacional.

1. Departamento de Ciencias de la Salud, Universidad Católica Santo Toribio de Mogrovejo. Lambayeque, Perú.

a. Docente. 


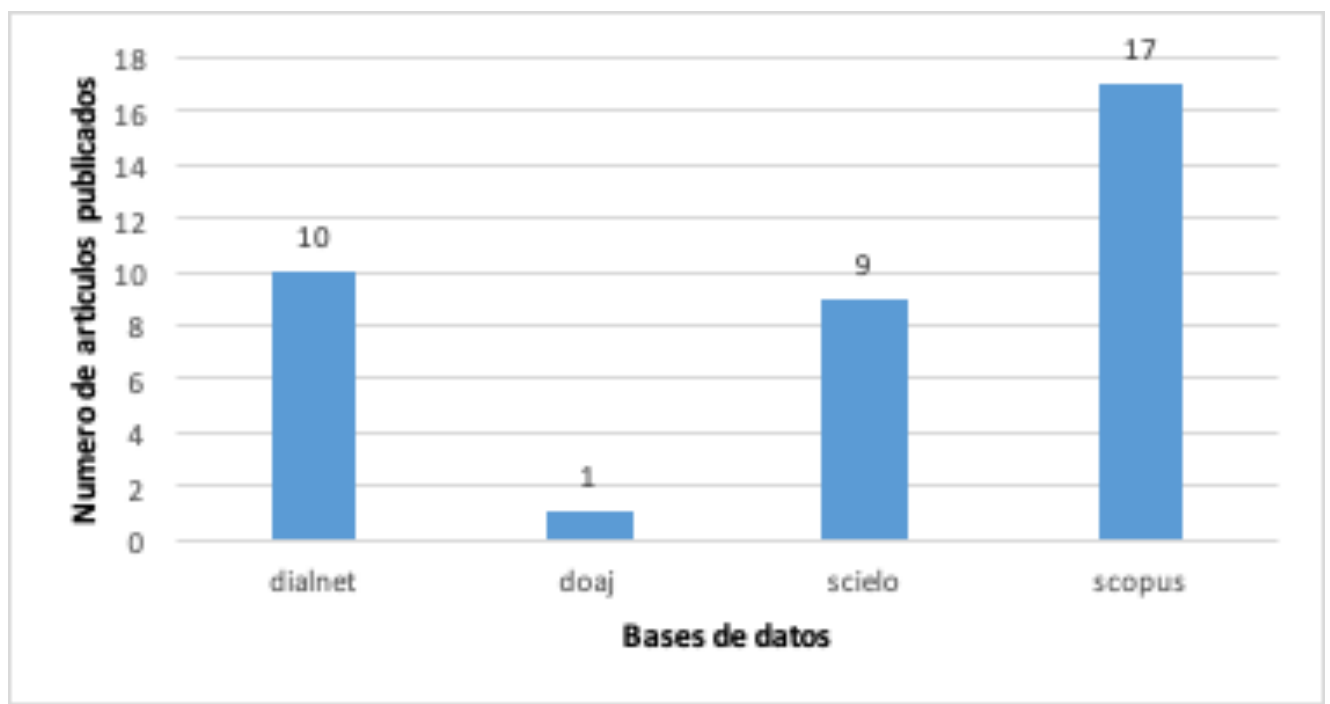

Gráfico 1. Distribución de los artículos científicos publicados en diversas bases de datos por los estudiantes de medicina de la Universidad Católica Santo Toribio de Mogrovejo, Lambayeque entre los años 2010 - 2019.

Es meritorio, por ejemplo, si comparamos las publicaciones del área Salud en base Scopus, en el año 2000 existían 314 artículos, y en el año 2018 el registro fue de 3290 , lo que es evidente que existe una inflexión en el crecimiento de las publicaciones peruanas a partir del 2014, fecha en que se aprobó la nueva ley universitaria (6).

Para la Escuela de Medicina Humana de la Universidad Católica Santo Toribio de Mogrovejo, Escuela Profesional acreditada por SINEACE y Universidad licenciada por la SUNEDU, constituye un aliciente y motivación para mejorar los procesos internos con el objetivo de incrementar, en número y en calidad, las publicaciones científicas que realizan los estudiantes. En el gráfico 1, se muestra nuestra producción científica con participación activa de los estudiantes de pregrado entre los años 2010 - 2019, la cual sin duda es una contribución al global que como país estamos mostrando a la comunidad científica internacional.

\section{REFERENCIAS BIBLIOGRÁFICAS}

1. León F. Pregrado en Medicina Humana: ¿Es posible graduarse publicando? Rev Med Hered. 2013; 24:175-177.

2. Isaza A, Botero H, Gonzales DC, Ospina LP, Velasco MM, Ocampo MP. Interés de los estudiantes de medicina por la investigación. CIMEL. 2010; 15 (1):9-13.

3. Torres F. Percepción de barreras y limitantes para realizar una tesis en pregrado en estudiantes de dos facultades de medicina humana de la región de Lambayeque en el periodo agosto-noviembre 2015 [tesis de pregrado]. Lima: Universidad San Martín de Porres; 2017.

4. Congreso de la República del Perú. Ley No 30220: Ley Universitaria. Lima: Congreso de la República; 2014.

5. Mayta P, Toro C, Alhuay J, Pacheco J. Scientific production and licensing of medical schools. Rev Peru Med Exp Salud Pública. 2019; 36(1):106-15.

6. Atamari-Anahui N, Roque-Roque J, Robles-Mendoza R, Nina-Moreno P, Falcón-Huancahuiri B. Publicación de tesis de pregrado en una facultad de Medicina en Cusco, Perú. Rev Med Hered. 2015; 26(4): 217-221. DOI: https://doi.org/10.20453/rmh. v26i4.2707. 